\title{
A NOVEL INTEGRATION OF PD-TYPE FUZZY LOGIC CONTROLLERS AND SMES DEVICES TO MAINTAIN THE NETWORK FREQUENCY OF A LARGE-SCALE POWER SYSTEM
}

\author{
VU DUY THUAN ${ }^{1, *}$, NGUYEN NGOC KHOAT ${ }^{1}$, HOANG NGOC NHAN $^{1}$, \\ THAI QUANG VINH ${ }^{2}$, NGO SI TAN ${ }^{2}$ \\ ${ }^{1}$ Faculty of Automation Technology, Electric Power University, Hanoi, Vietnam \\ ${ }^{2}$ Institute of Information Technology, Hanoi,Vietnam; *thuanvd@epu.edu.vn
}

\begin{abstract}
A modern electric power plant is typically considered to be a large-scale system. Due to continual and random occurrence of load changes, maintenance of network frequency (or load frequency control LFC) at its nominal value is one of the most crucial control problems in order to ensure the stability and reliability of such an electric power grid. This study investigates a new efficient integration of fuzzy logic controllers based on PD principle and superconducting magnetic energy storage (SMES) devices in an effort to protect the system frequency from the load variations. It is well known that the PD-based fuzzy logic controllers, when applied to an LFC strategy, are capable of damping quickly the oscillations of both the system frequency and tie-line power deviations. In addition, the load disturbances can be compensated if the network is applying the SMES devices. Therefore, the integration between them might become an efficiently feasible solution for the LFC issue. The superiority of the proposed control methodology over conventional regulators is verified through a number of numerical simulations which will be implemented in this study for a five-area electric power grid model.
\end{abstract}

Keywords. Large-scale power system, LFC, fuzzy logic controller, SMES devices, integrated control strategy.

\section{INTRODUCTION}

It is well known that an interconnected electric power grid has been generally considered to be a large-scale system which needs an efficiently overall control strategy in order to ensure the stable and reliable operation $[1,2,3,4,5]$. Such an interconnected power network usually works under constantly changing conditions of loads, which are dependent only on users, leading to a mismatch between real power and load demand, and thus creating the network frequency deviation from its nominal value. In addition to this phenomenon, tieline power bias might be automatically generated since it is directly proportional to the network frequency deviation. Both dynamic oscillations of the system frequency and tieline power flow must be damped in an efficient strategy to restore the normal operation of the electric power grid. Load frequency control (LFC) strategy to rapidly extinguish these dynamic deviations to satisfy acceptable tolerances has been carried out as a necessary part of automatic generation control (AGC) for a power system. If such an interconnected electric 
power grid is working under an efficient LFC methodology, its important control features can be successfully guaranteed $[3,6,7,8,9,10,11,12]$.

Each practical power plant normally contains a number of generating stations called control-areas. These areas are interconnected via transmission lines which are usually defined as tie-lines for power exchange. As a typical example of modern large-scale electric power systems, a five-area network model is chosen under study for this paper. The model is mathematically established first and then it is applied to test the feasibility of the LFC schemes. An efficient solution to the LFC problem applying intelligent controllers based on fuzzy logic technique is also proposed. This control strategy is dependent upon tie-line bias regulation, which was presented in $[1,3,8,11,12]$. One of the most important points when applying this control technique is that the tie-line power flow and system frequency deviations must be combined in a technical term called area control error (ACE). These ACE signals are considered to be the inputs of the LFC controllers and they must be driven to zero as rapidly as possible $[11,13,14,15,16]$. In this study, the PD (proportional-derivative)-based fuzzy logic controllers are chosen in dealing with the LFC issue. Therefore, both ACE and its derivative are fully used as the input signals in order to achieve the better main control characteristics, such as the smaller overshoots and the shorter settling times, compared with those of the conventional controllers using PI (proportional-plus-integral) regulators.

In this paper, superconducting magnetic energy storage (SMES) devices, which have been considered to design power system stabilizers, are also investigated to solve the problem of LFC $[17,18,19,20,21,22,23]$. It was found that these SMES units using thyristor-converters and superconducting coil are able to compensate for the sudden large load disturbances, such as the start and/or stop of big industrial factories. This is because their inductors can absorb or release active power in accordance with the decrease or increase of the loads in an electric power grid [12]. Therefore, such SMES units can be used in conjunction with efficient controllers to maintain the network frequency against load variations. The combination of these devices and PD-based fuzzy logic controllers when dealing with the LFC problem is one of the novel contributions of this study. In the following section of the present paper, the principle and model of an SMES unit will be discussed in detail. Then the third section mentions the modeling of a multi-area interconnected power system and particularly focuses on a five-area power network model as a typical case study. Load frequency controllers, including conventional PI and PD-based fuzzy logic regulators, will be described specifically in the fourth section of this paper. Finally, simulation results as well as conclusions will also be presented in the last two sections to verify the effectiveness of the proposed control strategy for a feasible solution of the LFC proposition.

\section{MODEL OF SUPERCONDUCTING MAGNETIC ENERGY STORAGE-SMES}

There seems to be no compelling reason to argue that an SMES unit is one of the most efficient devices for purpose of energy storage. Such an SMES unit can be considered to be a power system stabilizer since it is able to compensate for load variation, including sudden load changes. This puts forward the view that the SMES devices are quite able to be used in designing a robust LFC strategy.

Although there are generally three types of the SMES units, it is evident that SMES 
devices using thyristor converters might be suitably employed for the LFC. This is because they mainly regulate active power flow between AC and DC sides; thus they are able to directly impact on the network frequency, which is highly significant in the LFC problem. The illustration of a thyristor-based SMES unit is shown in Figure 1. In addition to a three-phase transformer, a six-pulse or twelve-pulse converter should be used in order to convert $\mathrm{AC}$ power flow into the DC counterpart. In Figure 1, a 12-pulse bridge AC/DC thyristor controlled converter is applied for such an SMES unit. In principle, such a 12pulse $\mathrm{AC} / \mathrm{DC}$ bridge converter contains two 6-pulse AC/DC converters in series (see Figure 1). This thyristor-based converter can typically act as a rectifier or an inverter depending upon the values of the firing angles $\alpha_{i}$, which are chosen by an electronic control system for the converter. In general, a DC voltage considered to be the output of this converter is theoretically the sum of two DC output voltages of two 6-pulse thyristor-based converters presented in Figure 1, and it should be given as

$$
\begin{aligned}
E_{d, i} & =U_{d 1}+U_{d 2} \\
& =V_{d 0} \cos \alpha+V_{d 0}^{\prime} \cos \alpha^{\prime}
\end{aligned}
$$

where $V_{d 0}$ and $V_{d 0}^{\prime}, \alpha$ and $\alpha^{\prime}$ denote the ideal no-load DC voltages and firing angles of converter $R_{1}$ and $R_{2}$, respectively. Assuming that such two 6-pulse AC/DC thyristor-based converters are completely symmetrical, the DC output of the 12-pulse converter can be calculated as follows [12]:

$$
E_{d, i}=2 V_{d, 0, i} \cos \alpha \text { with }\left\{\begin{array}{l}
E_{d, i}>0 \text { if } \alpha<\frac{\pi}{2} \text { in rectifying mode } \\
E_{d, i}<0 \text { if } \alpha>\frac{\pi}{2} \text { in inverting mode. }
\end{array}\right.
$$

In (2), $E_{d, i}, 2 V_{d 0, i}$ and $\alpha$ are DC output voltage (in volt), ideal no-load DC voltage (in volt) and firing angle (in degree) of the thyristor-based converter. One of the most important parts of an SMES device is a superconducting coil. In principle, applying such an inductor can assist the SMES device in absorbing power into or releasing from this unit. These two processes correspond to the charging or discharging of the coil, relating to the rectifier mode or inverter mode of the thyristor converter. In principle, there is a relation between the current and voltage of the superconducting coil as follows:

$$
L_{d, i}=\frac{1}{L_{d, i}} \int_{t_{0}}^{t} E_{d, i} d \tau+I_{d 0, i}
$$

where $I_{d 0, i}$ is the initial DC current through the coil. The real power value through the SMES can be computed as:

$$
P_{d, i}=E_{d, i} I_{d, i} .
$$

The energy which is stored in the coil should be calculated below:

$$
W_{d, i}=\int_{t_{0}}^{t} P_{d, i} d \tau+W_{d 0, i} .
$$

In terms of using the SMES as an efficient part of the LFC strategy, it is necessary to mathematically model such an SMES device. According to the tie-line bias control idea, 


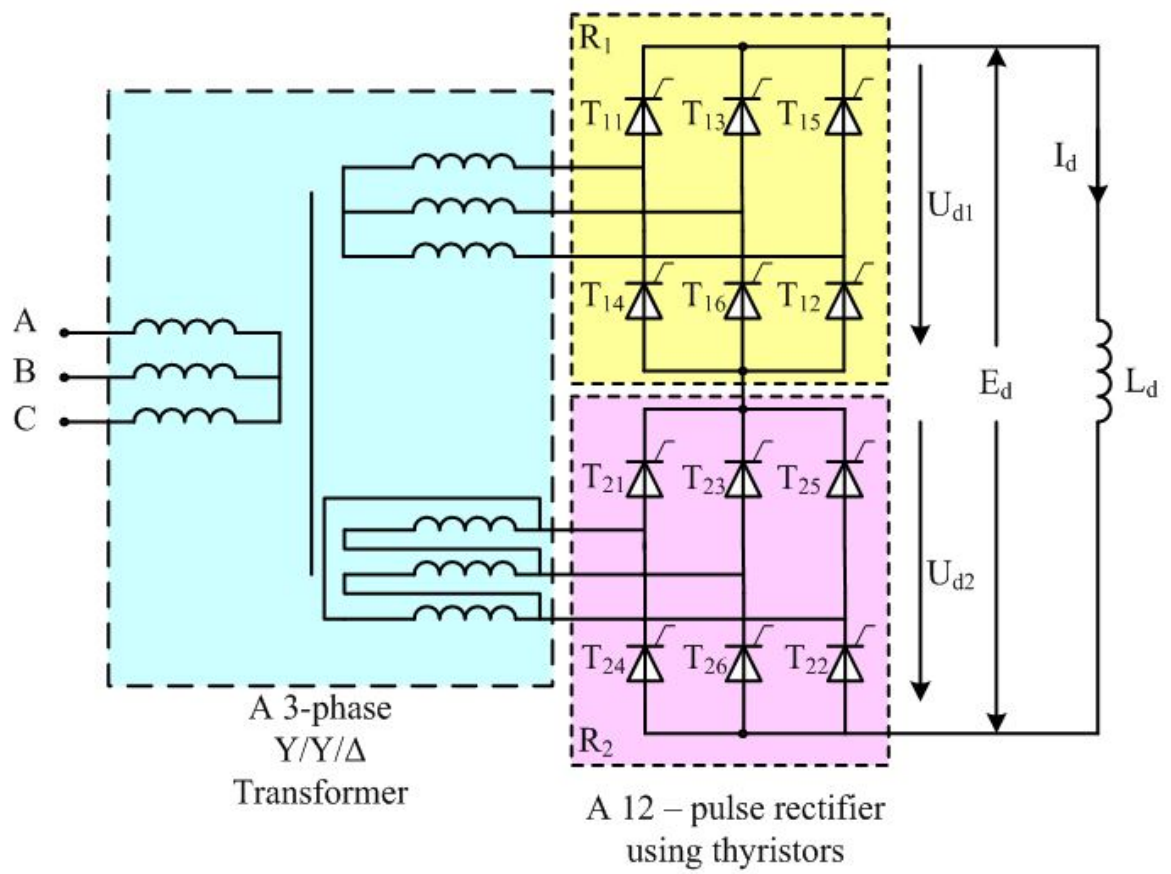

(a) Principle block diagram

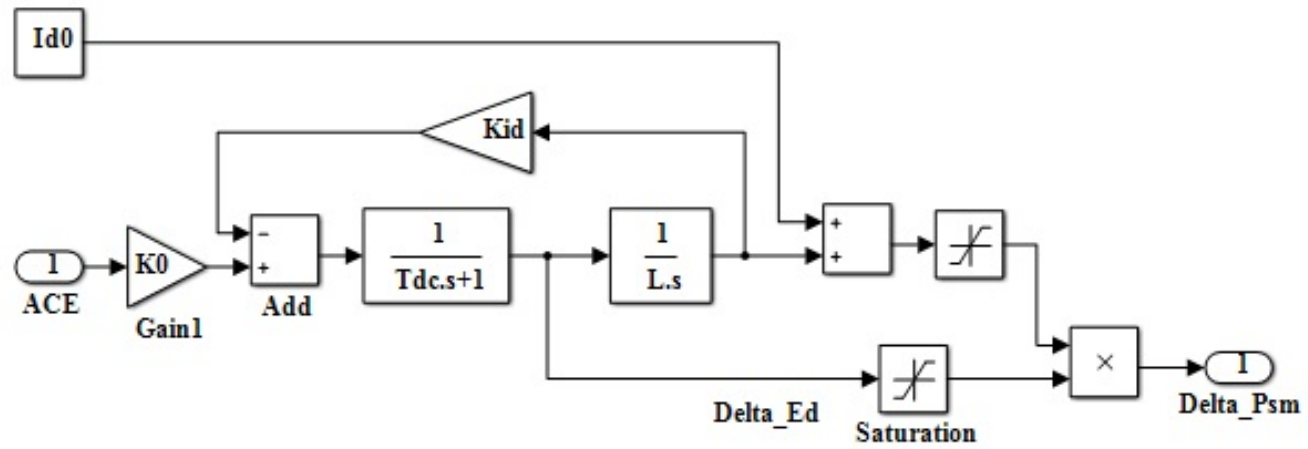

(b) SMES model built in MATLAB/Simulink platform for the LFC problem

Figure 1. Model of an SMES device using thyristor-based converters

ACE signals must be collected, and then they are taken to both the LFC regulator and the SMES. It means that each generating power should be equipped with an LFC controller and an SMES. The control idea is that the load changes can be compensated by charging or discharging of the inductor, thus the DC current $I_{d, i}$ should become a controlled quantity. According to [22], the increment of DC output voltage $E d, i$ can be computed as:

$$
\Delta E_{d, i}(s)=\frac{1}{1+s T_{d 0, i}}\left(K_{0, i} A C E_{i}(s)-K_{I d, i} \cdot \Delta I_{d, i}(s)\right) .
$$

Taking Laplace transform for equation (3), using a differential computation, the incre- 
ment of the DC current through the inductor is:

$$
\Delta I_{d, i}(s)=\frac{1}{s . L_{d, i}} \cdot \Delta E_{d, i}(s)
$$

where $K_{0, i}$ and $K_{I d, i}$ are constant factors of the SMES device. From (6) and (7), the mathematical model of this SMES built in MATLAB/Simulink platform is illustrated in Figure 1(b). The effectiveness of the SMES units for conducting the LFC problem will be presented in the following sections.

\section{MULTI-AREA INTERCONNECTED POWER SYSTEM MODEL}

There seems to be a consensus that modern electric power grids, treated as typical examples of large-scale systems, have highly complicated and various structures. In the present study, a typical example under scrutiny is a model of five-area interconnected power network. The fact is that there are also several structures regarding this model as shown in Figure 2. The available evidence seems to suggest that we might only need to choose a particular structure to verify the feasibility of the proposed control method. The others can be conducted in a same manner. From this point of view, the last structure presented in Figure 2(d) is selected in this study. As shown, the fifth control-area is supposed to be interconnected with the other ones for the power exchange. Assuming that the type of thermal power plant is under this study, each generation area or control-area from Figure 2(d) normally consists of three main parts in series: governor, non-reheat turbine and generatorload units (see Figure 3). When solving the LFC problem, the transfer functions of these units for the area $i$ can be found in $[4,5]$. It is straightforward to establish the model of the area $i$ in Laplace domain as follows:

$$
\begin{aligned}
& \Delta F_{i}(s)=\frac{1}{M_{i} . s+D_{i}}\left[\Delta P_{T, i}(s)-\Delta P_{D, i}(S)-\Delta P_{t i e, i}(s)\right], \\
& \Delta P_{T, i}(s)=\frac{1}{T_{T, i} \cdot s+1} \Delta P_{G, i}(s), \\
& \Delta P_{G, i} . s=\frac{1}{T_{G, i} \cdot s+1}\left[U_{i}(s)-\frac{1}{R_{i}} \Delta F_{i}(s)\right], \\
& \Delta P_{t i e, i}(s)= \begin{cases}\frac{2 \pi}{s} \sum_{j=1, j \neq i}^{5} T_{i j}\left[\Delta F_{i}(s)-\Delta F_{j}(s)\right] & \text { if there is tie-line } i-j \\
0, & \text { otherwise. }\end{cases}
\end{aligned}
$$

The meaning of the above variables can be found in $[17,3]$. A combination of the network frequency and tie-line power deviations can be executed through the following term:

$$
A C E_{i}(s)=\Delta P_{t i e, i}(s)+B_{i} \cdot \Delta F_{i}(s)
$$

where $B_{i}$ is a frequency bias factor. Taking the signal ACEi into the input signal of every LFC controller, it must be driven to zero according to the tie-line bias control method. There are also a number of LFC controllers which are able to use this signal for the control purpose satisfying acceptable control quality. The following section presents the discussion of several typical LFC controllers. 


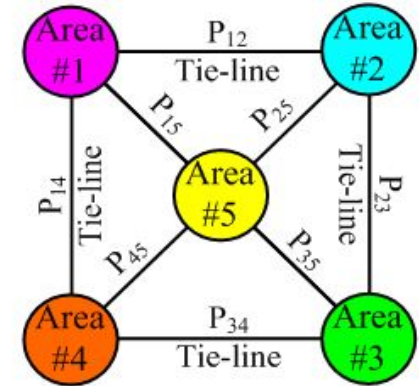

(a)

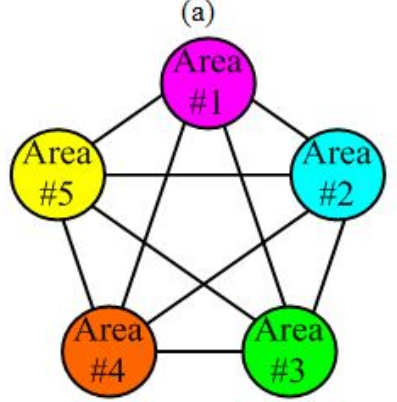

(c)

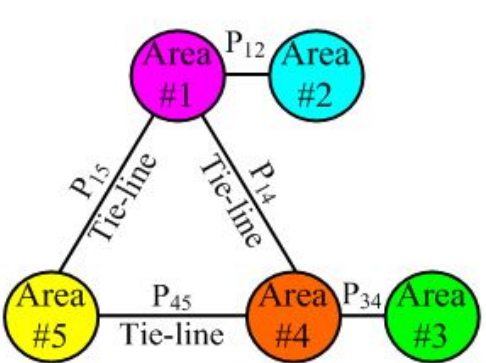

(b)

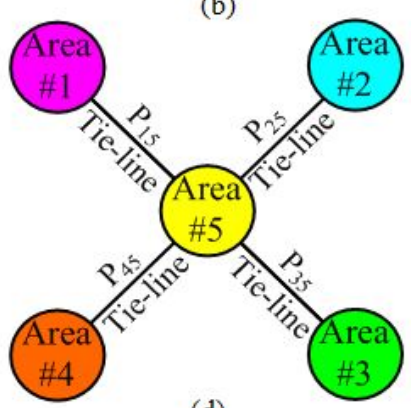

(d)

Figure 2. Simple models of five-area interconnected power systems

\section{DESIGN OF REGULATORS FOR LFC}

\subsection{Conventional LFC controllers}

In this section, the discussion will point to a number of conventional regulators used for the LFC issue. The fact is that three conventional regulators, including Integral, ProportionalIntegral (PI) and Proportional-Integral-Derivative (PID), have been employed for this aim. The first regulators (integral) with simple structure and easy tuning are difficult to ensure the high control performances, and thus their application is actually limited [8]. PID regulators can obtain the better control indices, however, due to a large number of tuning coefficients $\left(K_{P}, K_{I}\right.$ and $\left.K_{D}\right)$, it seems to be complicated to apply this type of load-frequency controller. In conclusion, the PI regulators should be chosen in the balance of the foregoing conditions. In principle, with the ACE signal used as the input, the control output of a PI regulator, which is taken to the governor, can be computed as follows:

$$
\left\{\begin{array}{l}
U_{i}(t)=K_{P, i} \cdot A C E_{i}(t)+K_{I, i} \int_{0}^{t} A C E_{i}(\tau) d \tau \\
U_{i}(s)=\left[K_{P, i}+K_{I, i} \frac{1}{s}\right] A C E_{i}(s),
\end{array}\right.
$$

where $K_{P, i}$ and $K_{I, i}$ denote the proportional gain and the integral gain of the above PI controller. The efficiency of the above PI regulators in dealing with the LFC problem will be presented in Section 5 of the paper.

\subsection{PD-based fuzzy logic controllers}

Fuzzy logic - based control strategy has been applied for a large number of control systems, especially for the nonlinear and uncertain control systems [16]. A large-scale power plant 
characterized by inherent nonlinearities and uncertainties is appropriate to the application of fuzzy logic controllers. Although there have been numerous studies on this topic, LFC methodology based on fuzzy logic technique is still of great interest to researchers. This paper aims to represent the design of a typical fuzzy logic - based LFC strategy in combination with the SMES devices as mentioned earlier in order to achieve excellent control performances. The proposed fuzzy logic architecture is illustrated in Figure 3, including two inputs $\left(A C E_{i}\right.$ and derivative of $\left.A C E_{i}\right)$ and one output $\left(U_{i}\right)$. In general, each fuzzy logic inference system contains three parts, i.e., fuzzification, rule base and defuzzification. The fundamental knowledge of a fuzzy logic architecture can be found clearly in [16]. The fuzzy logic model used in this work is called the PD-based fuzzy logic controller. The principle of this fuzzy logic inference system can be deduced from Figure 3 as follows:

$$
U_{i}(t)=K_{u} \cdot u_{i}(t)=K_{u}\left[K_{e} A C E_{i}(t) d t+K_{d e} \frac{d}{d t} A C E_{i}(t)\right] .
$$

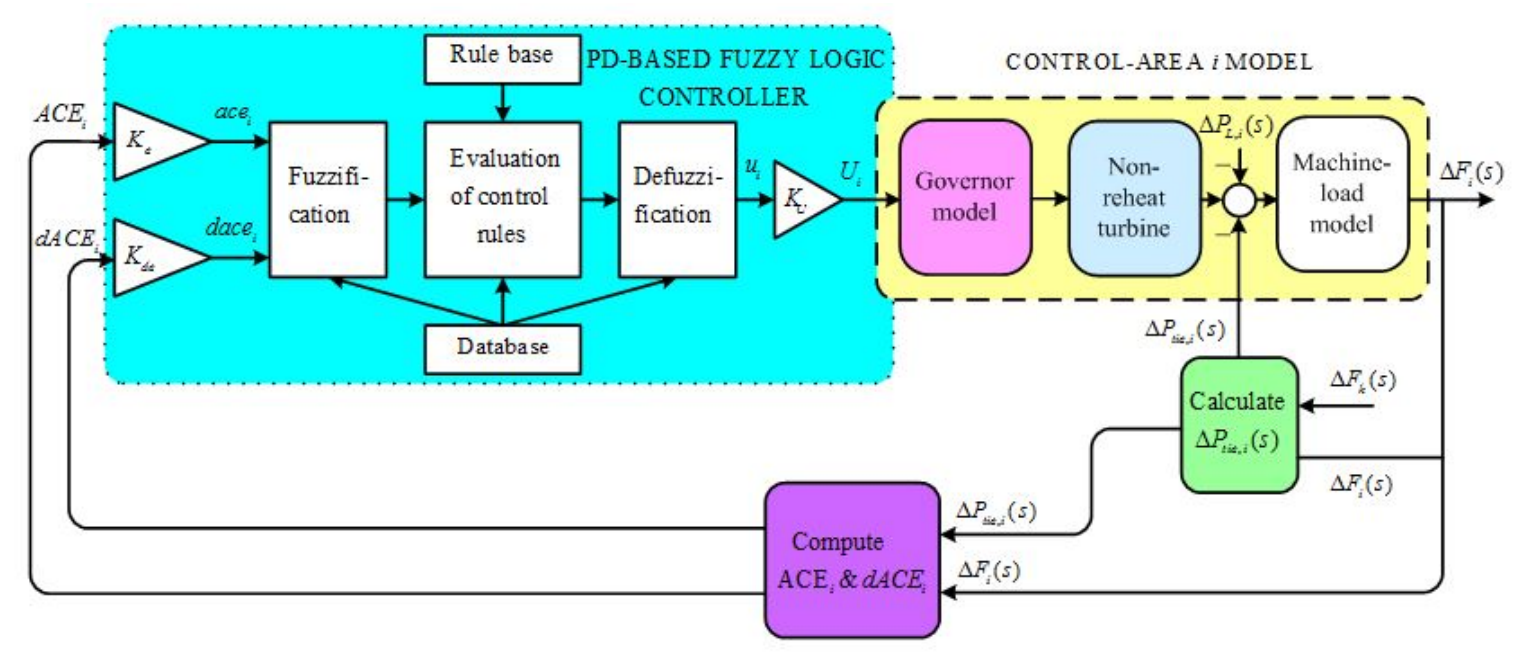

Figure 3. Structure of a PD-based FL controller for the area $i$

In (14), three factors, $K_{e}, K_{d e}$ and $K_{u}$ are defined as three tuning factors of the fuzzy logic architecture corresponding to $A C E_{i}$, derivative of $A C E_{i}$ and output signal $U_{i}$. From (14), it is straightforward to yield the following:

$$
U_{i}(t)=K_{p} A C E_{i}(t)+K_{D} \frac{d}{d t} A C E_{i}(t)
$$

where $K_{p}=K_{U} \cdot K_{e}$ and $K_{D}=K_{U} \cdot K_{d e}$. The consensus view seems to be that these two coefficients correspond to two factors of a PD controller, which need to be tuned when designing a regulator based on PD principle. In other words, it might be defined as a PDlike fuzzy logic controller.

To design a fuzzy logic based controller, four steps need to be executed as follows:

Step 1: Determine the number and types of membership functions. In this study, seven membership functions are chosen for each input: $E_{k}(k=1,2, \ldots, 7)$ for ace $_{i}(t)$ and 
Table 1. Rule matrix for the PD-based FL controller

\begin{tabular}{|c|c|c|c|c|c|c|c|}
\hline \multirow{2}{*}{$\boldsymbol{a c e}_{\boldsymbol{i}}(\boldsymbol{t})$} & \multicolumn{7}{|c|}{$\boldsymbol{d a c e}_{\boldsymbol{i}}(\boldsymbol{t})$} \\
\cline { 2 - 8 } & $\boldsymbol{D E}_{\mathbf{1}}$ & $\boldsymbol{D E}_{\mathbf{2}}$ & $\boldsymbol{D E}_{\mathbf{3}}$ & $\boldsymbol{D E}_{\mathbf{4}}$ & $\boldsymbol{D E}_{\mathbf{5}}$ & $\boldsymbol{D E}_{\mathbf{6}}$ & $\boldsymbol{D E}_{\mathbf{7}}$ \\
\hline $\boldsymbol{E}_{1}$ & $U_{1}$ & $U_{1}$ & $U_{1}$ & $U_{2}$ & $U_{3}$ & $U_{4}$ & $U_{5}$ \\
\hline $\boldsymbol{E}_{2}$ & $U_{1}$ & $U_{1}$ & $U_{2}$ & $U_{3}$ & $U_{4}$ & $U_{5}$ & $U_{6}$ \\
\hline $\boldsymbol{E}_{3}$ & $U_{1}$ & $U_{2}$ & $U_{3}$ & $U_{4}$ & $U_{5}$ & $U_{6}$ & $U_{7}$ \\
\hline $\boldsymbol{E}_{4}$ & $U_{2}$ & $U_{3}$ & $U_{4}$ & $U_{5}$ & $U_{6}$ & $U_{7}$ & $U_{8}$ \\
\hline $\boldsymbol{E}_{5}$ & $U_{3}$ & $U_{4}$ & $U_{5}$ & $U_{6}$ & $U_{7}$ & $U_{8}$ & $U_{9}$ \\
\hline $\boldsymbol{E}_{6}$ & $U_{4}$ & $U_{5}$ & $U_{6}$ & $U_{7}$ & $U_{8}$ & $U_{9}$ & $U_{9}$ \\
\hline $\boldsymbol{E}_{7}$ & $U_{5}$ & $U_{6}$ & $U_{7}$ & $U_{8}$ & $U_{9}$ & $U_{9}$ & $U_{9}$ \\
\hline
\end{tabular}

$D E_{l}(l=1,2, \ldots, 7)$ for dace $_{i}(t)$. All of these membership functions are defined as symmetrical triangular functions. In addition, nine Gaussian curve membership functions are selected for the output of the proposed PD-type fuzzy logic model, including $U_{1}, U_{2}, \ldots, U_{9}$ (see Figure 4).

Step 2: Determine rule base of the fuzzy logic architecture. The rule base is normally defined depending on experience of the experts. We propose a rule base matrix indicated in Table 1 for the PD-like fuzzy logic model based on genetic algorithm (presented in $[17,11]$ ). Because of the continuous and random load change, in order to ensure high quality of the load-frequency control system, it is necessary to use a sufficient number of membership functions. It means that the input signals as well as the output signal need to be divided into small parts to improve the control performances of the system. Normally, the number of membership functions when applying PD-like fuzzy logic architecture is from three to ten ones. In this work, as presented in Step 1, seven membership functions are chosen for each input $\left(a_{c e}(t)\right.$ and dace $\left.(t)\right): E_{1}, E_{2}, \ldots, E_{7}$ for $a c e_{i}(t)$ and $D E_{1}, D E_{2}, \ldots, D E_{7}$ for dace $e_{i}(t)$. Besides, in order to enhance control capability for the load-frequency regulator presented in this study, we selected nine membership functions for the output signal: $U_{1}, U_{2}, \ldots, U_{9}$. Using these membership functions for the fuzzy logic reasoning system, it is highly reliable to make sure that the output signal $(\Delta f(t), A C E(t))$ can be driven to zero as quickly as possible. Some explanations in "if-then" form might be drawn from Table 1 as follows:

(i) if $a c e_{i}(t)$ and dace $_{i}(t)$ are equal to zeros or very near to zeros, then the current state of output should be remained;

(ii) if $a c e_{i}(t)$ is not zero however it might be slightly changing, then it is necessary to maintain the current control state;

(iii) if $a c e_{i}(t)$ is varying significantly, then we need modify the control signal based on the magnitude and sign of $a c e_{i}(t)$ and $d_{a c e}(t)$ to force $a c e_{i}(t)$ towards zero.

Step 3: Choose the method to defuzzification process. In this study, max-MIN rule is selected for this aim since this rule might be of popularity and high efficiency.

Step 4: Compute the scaling factors of this fuzzy logic model. As mentioned earlier, three scaling factors, including $K_{e}, K_{d e}$ and $K_{u}$ might need to be efficiently tuned in order to obtain the control performances with high quality. For this study, we have used "try-anderror" method to the determination of these three factors. 
The above four steps to design a LFC strategy will be implemented in combination with the application of the SMES devices (presented in Section 2). The efficiency of the proposed control methodology will be demonstrated in the following section.
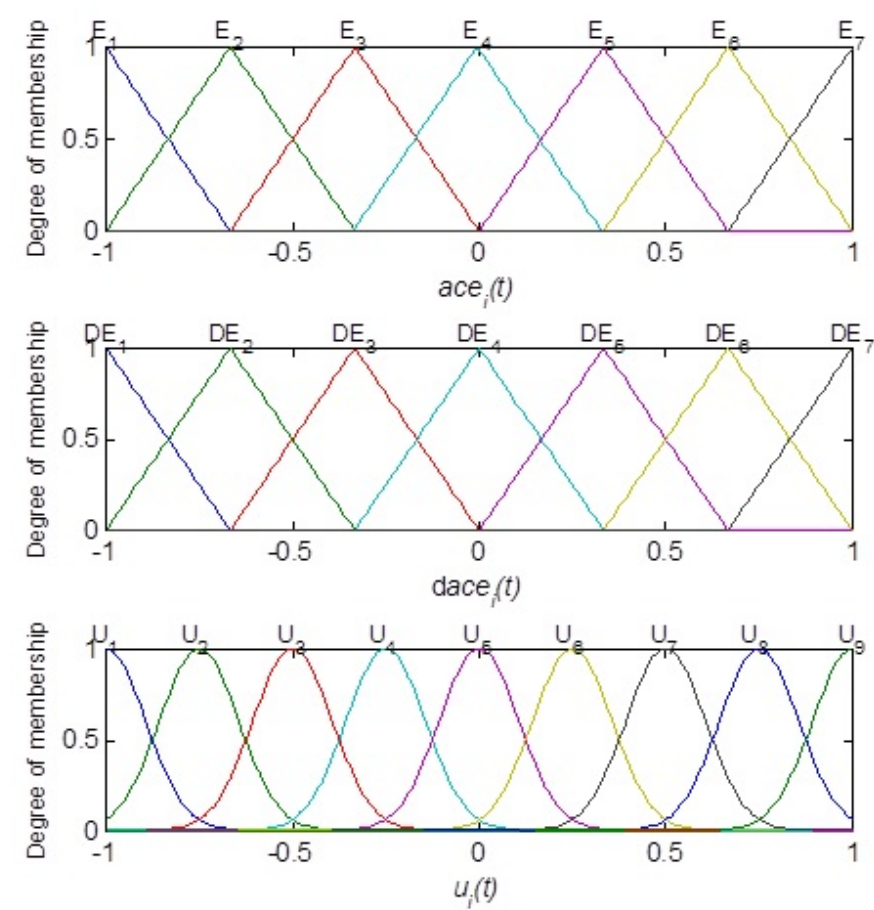

Figure 4. Illustration of membership functions for two inputs and one output of the proposed fuzzy logic architecture

\section{NUMERICAL SIMULATIONS AND DISCUSSIONS}

In this section, numerical simulations will be implemented to verify the effectiveness of the proposed controllers as well as the SMES devices to solve the LFC problem. The overall simulation model is built in MATLAB/Simulink package as shown in Figure 5 for the case study mentioned earlier (see Figure 2(d)).

Since loads in a power system can vary randomly at any generating station, loadfrequency controllers have to respond efficiently enough to restore the steady state of the network as rapidly as possible. In this paper, two cases of load variations are to be considered as follows:

(i) Case 1: Let load variation only appear randomly in the most important generating station (area 5) as shown in Figure 6(a). In this case, the step-load will increase at instants of $0(\mathrm{sec}), 5(\mathrm{sec})$ and $10(\mathrm{sec})$ with the corresponding magnitudes of 0.005 (p.u.), 0.01(p.u.) and 0.02(p.u.). Such step-load will decrease at instants of $15(\mathrm{sec})$ and 20 (sec) to the amplitudes of 0.01 (p.u.) and 0.05(p.u.), respectively. It would be consistent with an actual condition of daily load in a power plant. 


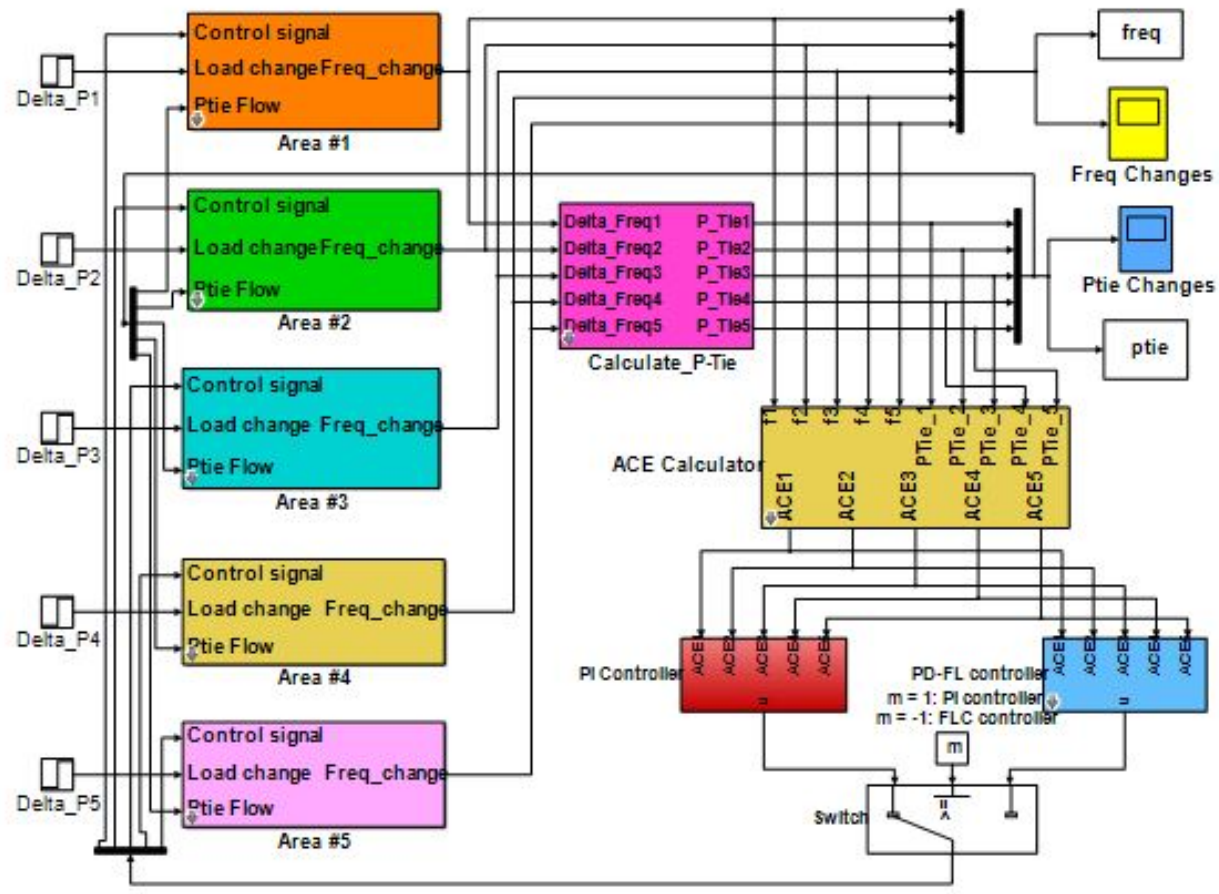

(a) Simulation model with subsystems

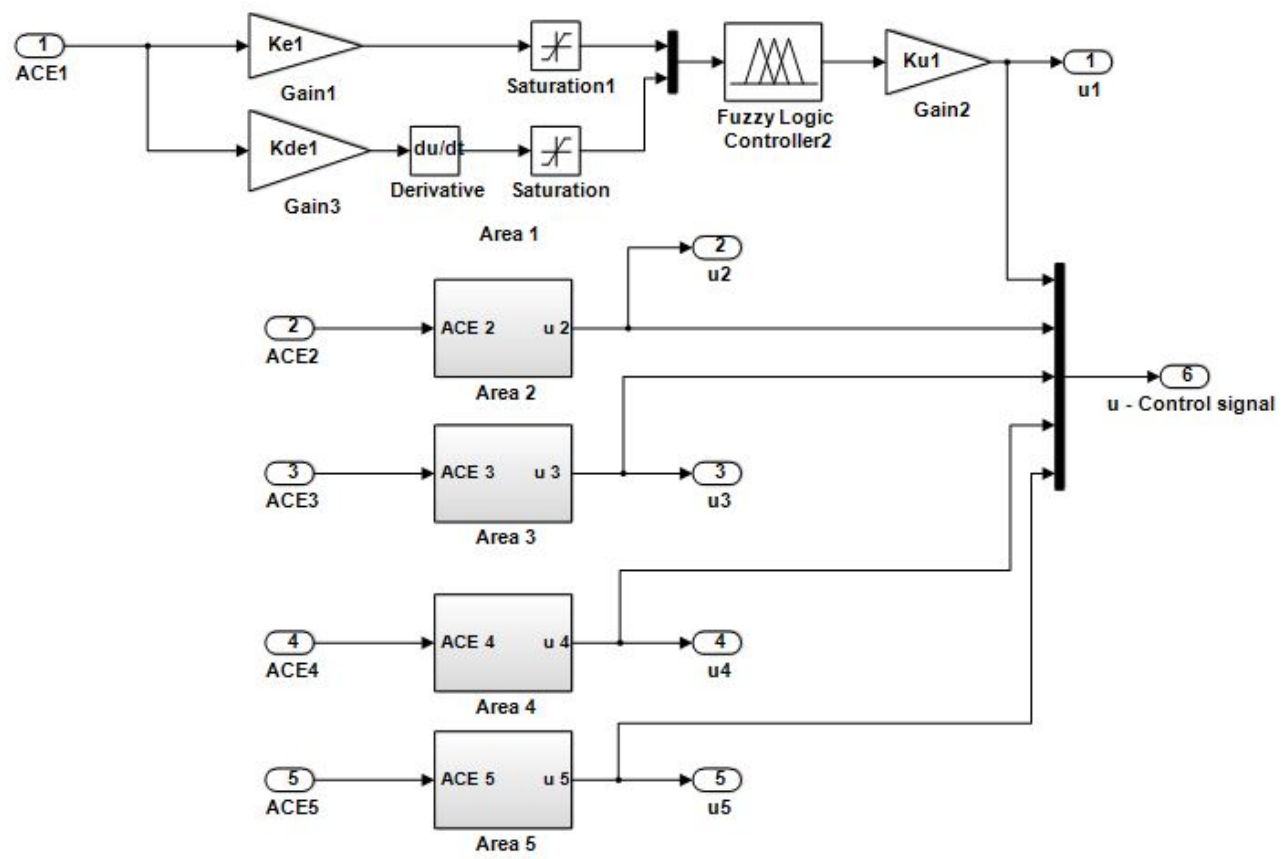

(b) Details of the subsystem "PD-FL controller"

Figure 5. Simulation model using different controllers built in MATLAB/Simulink platform 


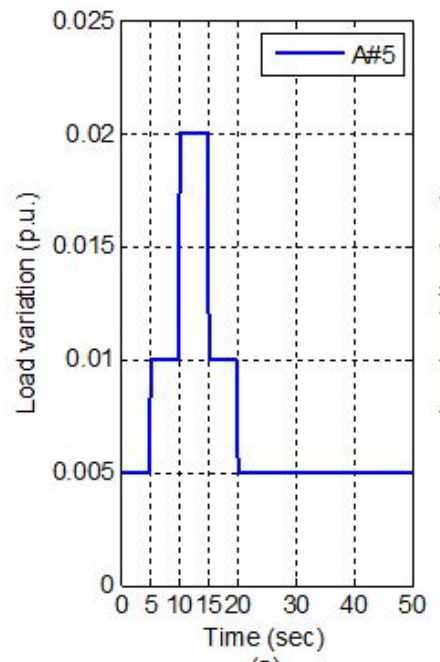

(a)

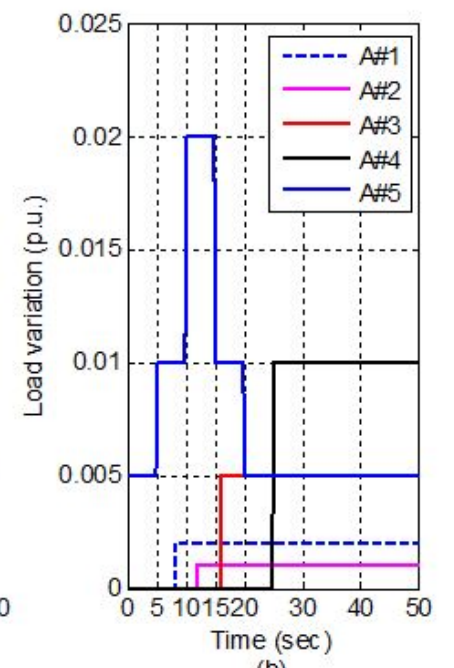

(b)

Figure 6. Two load changes applied for simulation purpose

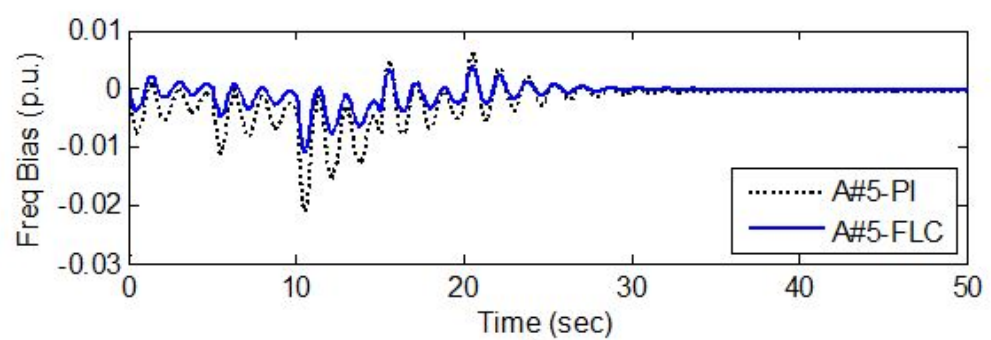

(a)

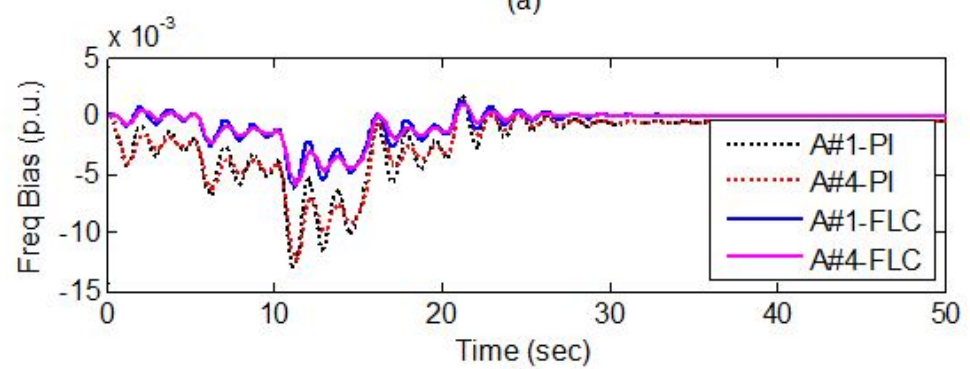

(b)

Figure 7 . Frequency deviations in the areas \#1, \#4 and \#5 in the first simulation case

(ii) Case 2: Let load variations occur at all generating areas in the power system model built earlier as shown in Figure 6(b). Load in the area \#5 is the same of the first case, meanwhile loads in the other areas are embedded at different instants and amplitudes.

In the above two simulation cases, the SMES units will also be applied to validate their performances. Simulation results obtained are illustrated in Figures 7-10 as well as Table 2. The following discussions will demonstrate the dominant performances of the controllers studied in this work. 


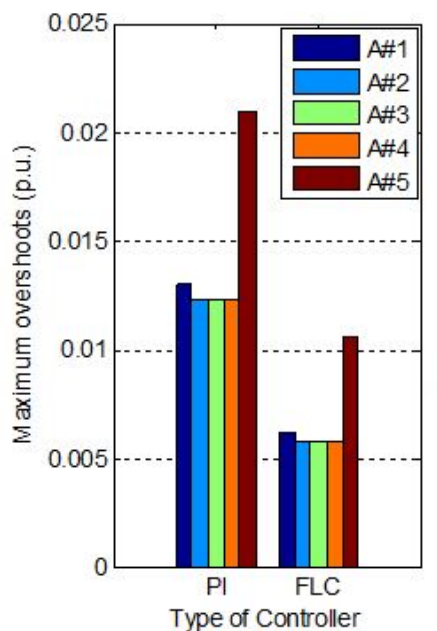

(a)

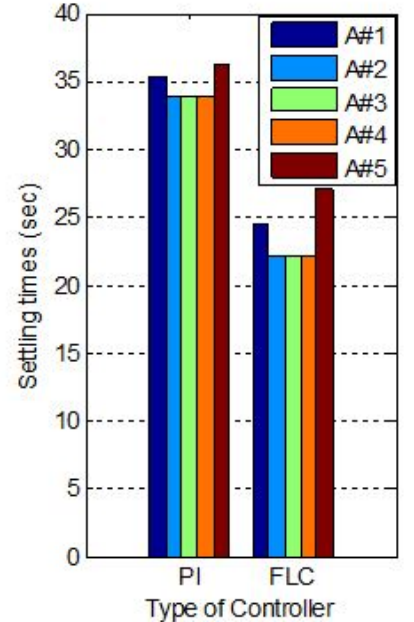

(b)

Figure 8. A comparison of maximum overshoots (absolute values) and settling times for all areas in the first simulation case

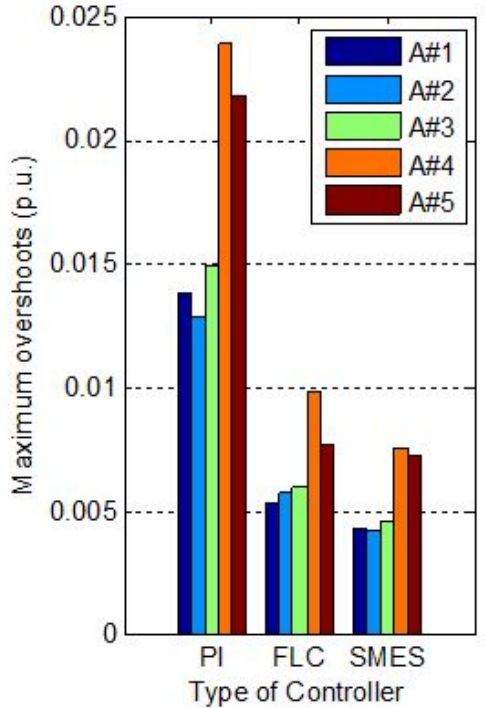

(a)

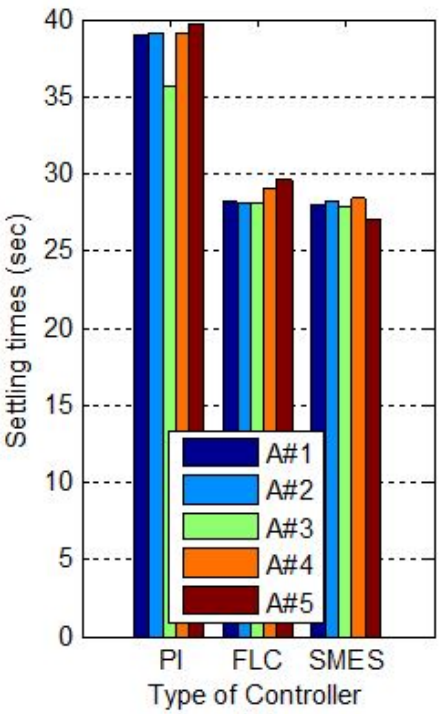

(b)

Figure 9. A comparison of (a) maximum overshoots (absolute values) and (b) settling times for all areas in the second simulation case

\subsection{Capability of PD-based fuzzy logic controllers}

In the first simulation case, Figure 7 describes the response of frequency deviations in several areas using PI and PD-based fuzzy logic controllers. To illustrate numerical results, Figure 8 shows a comparison of maximum overshoots (absolute values) and settling times for all areas with a desired frequency tolerance of $0.05 \%$. From these results, it is clear to draw the following conclusions: 


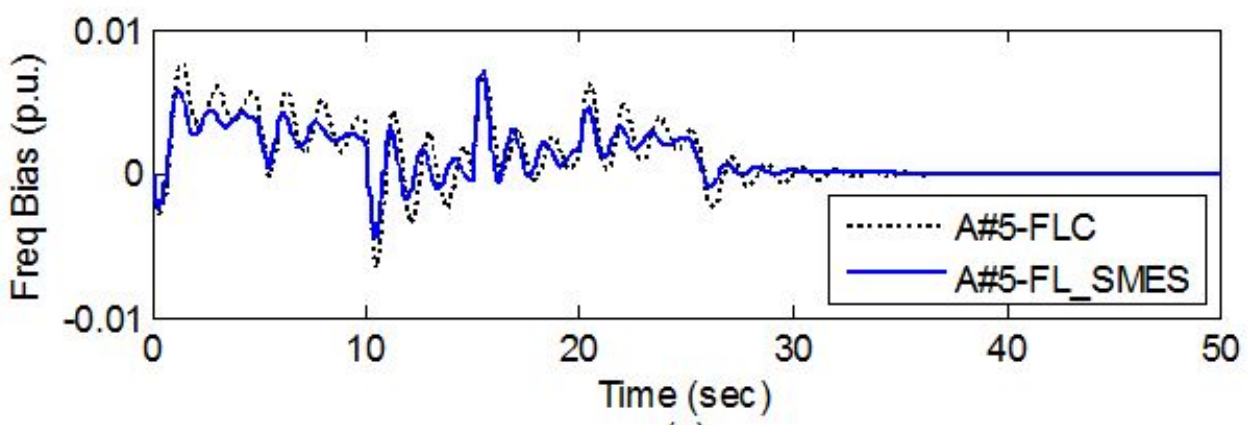

(a)

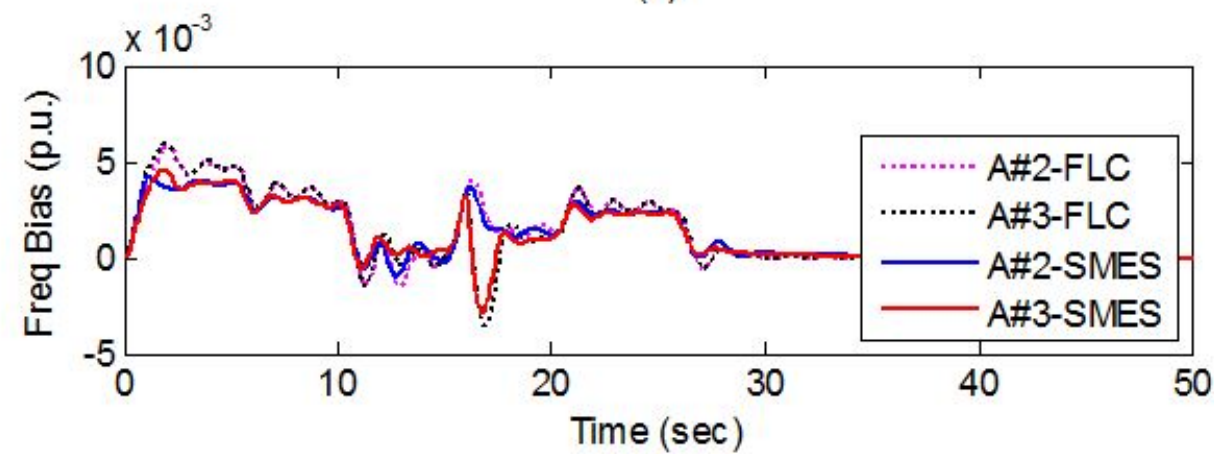

(b)

Figure 10. Frequency deviations in three areas 2, 3 and 5 in the second simulation case

(1) The maximum overshoots as well as the settling times of PD-based fuzzy logic controllers are much smaller than PI regulators. As a result, the control performances of the proposed controllers are much better than those of the conventional PI regulators for conducting the maintenance of the network frequency against the load disturbances.

(2) Since load change only appears in the fifth area, the frequency of this station will be affected with the biggest deviation (the highest overshoot and the longest settling time). It means that the control indexes in this area are at the worst.

Table 2. A comparison of maximum overshoots (absolute values) of tie-line power deviations, in p.u., for all of areas in both simulation cases

\begin{tabular}{|l|c|c|c|c|c|c|}
\hline \multirow{2}{*}{ Area } & \multicolumn{3}{|c|}{ Case 1 } & \multicolumn{3}{c|}{ Case 2 } \\
\cline { 2 - 7 } & PI & PD-FL & FL-SMES & PI & PD-FL & FL-SMES \\
\hline Area 1 & 0.0048 & 0.0028 & 0.0021 & 0.0035 & 0.0019 & 0.0015 \\
\hline Area 2 & 0.0049 & 0.0029 & 0.0022 & 0.0051 & 0.0030 & 0.0024 \\
\hline Area 3 & 0.0049 & 0.0029 & 0.0020 & 0.0051 & 0.0033 & 0.0024 \\
\hline Area 4 & 0.0049 & 0.0029 & 0.0023 & 0.0053 & 0.0033 & 0.0028 \\
\hline Area 5 & 0.0195 & 0.0114 & 0.0090 & 0.0189 & 0.0114 & 0.0087 \\
\hline
\end{tabular}

Considering the second simulation case, the results obtained are shown in Figure 9 and Table 2. It is very similar to the first simulation case to assert that the proposed fuzzy 
logic controllers have been achieved the better control performances compared with the conventional PI regulators.

\subsection{Effectiveness of the SMES devices}

In order to verify the performance of the SMES devices, Figure 9, Figure 10 and Table 2 represent simulation results obtained when using PD-based fuzzy logic controllers with and without SMES units. Figure 9 illustrates a comparison of maximum overshoots and settling times of frequency biases for all areas in the second simulation case. Meanwhile, Figure 10 shows the response of frequency changes in the areas 2, 3 and 5 only for controllers based on fuzzy logic with and without the SMES units. Also, Table 2 presents the tie-line power flow deviations for all of areas in two simulation cases using different controllers. From these results, it is confirmed that the combination of the fuzzy logic architecture and the SMES devices can obtain the outstanding control performances compared with the other two control methods when maintaining the system frequency after occurrence of load disturbances in an electric power plant. Thus, it is the demonstration for the feasibility of the proposed control methodology.

\section{CONCLUSIONS}

This work concentrates on solving the LFC problem in a multi-area interconnected power plant, which has been considered to be a typical example of large-scale systems. A particular model of five-area power networks has been designed as a case study of the practically complicated electric grids. Furthermore, SMES devices and load-frequency controllers based on tie-line bias control scheme are investigated in order to stabilize the network frequency as well as the tie-line power flow against load variations. From this study, it is well known the following conclusions can be drawn.

(1) LFC is the core of an automatic generation control, playing an important role to ensure the stability and economy of a practical large-scale interconnected power plant.

(2) PD-based fuzzy logic controllers can solve efficiently the issue of LFC, achieving the better control performances compared with conventional regulators, such as PI.

(3) SMES units can be used in combination with efficient controllers to obtain the optimal control properties, such as the lowest overshoots and the smallest settling times.

(4) Due to the complexity of a large and modern power system in practice, it is necessary to model more exactly such electric grid to deal with LFC problem by applying the improved control strategies as mentioned in this study.

Finally, since loads of a power system, which depend only on customers, can vary randomly and continually over time, they should be measured to create the fully practical database for necessary control schemes. This might be of interest for our work in the future. 


\section{APPENDICES}

\section{A Nomenclature}

$i \quad$ index of control-area $i, i=1,2, n$

$E d, i \quad$ DC output voltage of the AC/DC thyristor based converter, $V$

$\Delta E_{d, i}(s)$ increment of $E_{d, i}, V$

$V_{d 0, i} \quad$ ideal no-load DC voltage of a 6 -pulse AC/DC bridge converter, $V$

$\alpha \quad$ firing angle of thyristor, degree

$I_{d, i} \quad$ DC current through the superconducting coil, A

$I_{d 0, i} \quad$ initial DC current through the superconducting coil, A

$\Delta I_{d, i}(s) \quad$ increment of the DC current through the superconducting coil, A

$L_{d, i} \quad$ inductance value of the superconducting coil, $\mathrm{H}$

$P_{d, i} \quad$ real DC output power of the superconducting coil, W

$\Delta P_{d, i} \quad$ change of real DC output power of the superconducting coil, $\mathrm{W}$

$W_{d, i} \quad$ stored energy in the coil, $\mathrm{J}$

$W_{d 0, i} \quad$ initial stored energy in the coil, $\mathrm{J}$

$T_{d c, i} \quad$ time delay of the converter in the power conditioning unit, $\mathrm{s}$

$K_{0, i} \quad$ gain corresponding to ACE input

$K_{I d, i} \quad$ gain corresponding to $\Delta I_{d, i}$ input

$f_{i} \quad$ real network frequency, $H_{z}$

$f_{n} \quad$ nominal network frequency, $f_{n}=50 \mathrm{~Hz}$

$\Delta F_{i}(s) \quad$ frequency deviation of area $i, p . u$.

$\Delta P_{D, i}(s) \quad$ load variation, $p . u$.

$T_{G, i} \quad$ time constant of governor, sec

$T_{T, i} \quad$ time constant of non-reheat turbine, sec

$M_{i} \quad$ generator inertia constant, $p . u$.

$D_{i} \quad$ load damping factor, p.u. $M W / H z$

$T_{i j} \quad$ tie-line time constant, sec

$P_{\text {tie }, i}(s) \quad$ tie-line power flow, p.u.

$\Delta P_{t i e, i}(s)$ deviation of tie-line power flow, $p . u$.

$B_{i} \quad$ frequency bias factor, $\mathrm{MW} / \mathrm{p} \cdot \mathrm{u} \cdot \mathrm{Hz}$

$R_{i} \quad$ speed regulation, $H z / M W$

$\Delta_{P T, i}(s) \quad$ change of output of turbine

$\Delta P_{G, i}(s) \quad$ change of output of governor

$U_{i}(s) \quad$ control signal to governor

B. Parameters of the five-area electric power system model used for numerical simulations

$$
\begin{aligned}
& T_{G, 1}=0.08, T_{G, 2}=0.12, T_{G, 3}=T_{G, 4}=T_{G, 5}=0.10 \\
& T_{T, 1}=T_{T, 2}=0.35, T_{T, 3}=T_{T, 4}=T_{T, 5}=0.30 \\
& K_{P, 1}=120, K_{P, 2}=98, K_{P, 3}=K_{P, 4}=K_{P, 5}=105 \\
& T_{P, 1}=18, T_{P, 2}=20, T_{P, 3}=T_{P, 4}=T_{P, 5}=15, T_{i j}=\frac{1}{\sqrt{2}} .
\end{aligned}
$$




\section{REFERENCES}

[1] H. Bevrani and T. Hiyama, Intelligent automatic generation control. CRC press New York, 2011.

[2] R. C. Dorf and R. H. Bishop, Modern control systems. Pearson (Addison-Wesley), 1998.

[3] D. Ernst, M. Glavic, and L. Wehenkel, "Power systems stability control: Reinforcement learning framework," IEEE transactions on power systems, vol. 19, no. 1, pp. 427-435, 2004.

[4] T. Q. Vinh, H. M. Dao, and H. S. Bang, "Decentralized stabilization of complex systems by combination of conventional and fuzzy controls," International Journal of Uncertainty, Fuzziness and Knowledge-Based Systems, vol. 7, no. 04, pp. 423-427, 1999.

[5] T. Q. Vinh and K. Hirota, "Decentralized robust fuzzy sliding mode control design of interconnected uncertain systems," J. of Advanced Computational Intelligence, vol. 6, no. 1, pp. 56-61, 2002.

[6] M. Chandrashekar and R. Jayapal, "Design and comparison of i, pi, pid and fuzzy logic controller on agc deregulated power system with hvdc link," in Circuits, Controls and Communications (CCUBE), 2013 International conference on. IEEE, 2013, pp. 1-6.

[7] D. Devaraj and B. Selvabala, "Real-coded genetic algorithm and fuzzy logic approach for realtime tuning of proportional-integral-derivative controller in automatic voltage regulator system," IET generation, transmission \& distribution, vol. 3, no. 7, pp. 641-649, 2009.

[8] N.-K. Nguyen, Q. Huang, and T.-M.-P. Dao, "An evaluation of different controllers based on the network frequency maintenance strategy for a large and multi-control-area interconnected power system," Electric Power Components and Systems, vol. 43, no. 11, pp. 1257-1267, 2015.

[9] P. Subbaraj and K. Manickavasagam, "Generation control of interconnected power systems using computational intelligence techniques," IET Generation, Transmission 83 Distribution, vol. 1, no. 4, pp. 557-563, 2007.

[10] A. K. Thirukkovulur, H. Nandagopal, and V. Parivallal, "Decentralized control of multi-area power system restructuring for lfc optimization," in Computational Intelligence $\&$ Computing Research (ICCIC), 2012 IEEE International Conference on. IEEE, 2012, pp. 1-6.

[11] R. Verma, S. Pal et al., "Intelligent automatic generation control of two-area hydrothermal power system using ann and fuzzy logic," in Communication Systems and Network Technologies (CSNT), 2013 International Conference on. IEEE, 2013, pp. 552-556.

[12] T.-M.-P. Dao, Y. Wang, and N.-K. Nguyen, "Novel hybrid load-frequency controller applying artificial intelligence techniques integrated with superconducting magnetic energy storage devices for an interconnected electric power grid," Arabian Journal for Science and Engineering, vol. 41, no. 9, pp. 3309-3320, 2016.

[13] C. H. Lee, F. Y. Chang, and C. M. Lin, "An efficient interval type-2 fuzzy cmac for chaos timeseries prediction and synchronization," IEEE Transactions on Cybernetics, vol. 44, no. 3, pp. 329-341, March 2014.

[14] Y. Li, S. Tong, Y. Liu, and T. Li, "Adaptive fuzzy robust output feedback control of nonlinear systems with unknown dead zones based on a small-gain approach," IEEE Transactions on Fuzzy Systems, vol. 22, no. 1, pp. 164-176, 2014. 
[15] G. Panda, S. Panda, and C. Ardil, "Hybrid neuro fuzzy approach for automatic generation control of two-area interconnected power system," International Journal of Computational Intelligence, vol. 5, no. 1, pp. 80-84, 2009.

[16] T. J. Ross, Fuzzy logic with engineering applications. John Wiley \& Sons, 2009.

[17] H. Bevrani, Robust power system frequency control. Springer, 2009, vol. 85.

[18] P. Khayyer and U. Ozguner, "Decentralized control of large-scale storage-based renewable energy systems," IEEE Transactions on Smart Grid, vol. 5, no. 3, pp. 1300-1307, 2014.

[19] I. Ngamroo, "An optimization technique of robust load frequency stabilizer for superconducting magnetic energy storage," Energy conversion and management, vol. 46, no. 18, pp. 3060-3090, 2005 .

[20] S. Sridhar and M. Govindarasu, "Model-based attack detection and mitigation for automatic generation control," IEEE Transactions on Smart Grid, vol. 5, no. 2, pp. 580-591, 2014.

[21] Y. Wan and J. Zhao, "H control of single-machine infinite bus power systems with superconducting magnetic energy storage based on energy-shaping and backstepping," IET Control Theory E) Applications, vol. 7, no. 5, pp. 757-764, 2013.

[22] A. S. Yunus, A. Abu-Siada, and M. Masoum, "Application of smes unit to improve dfig power dispatch and dynamic performance during intermittent misfire and fire-through faults," IEEE Transactions on Applied Superconductivity, vol. 23, no. 4, pp. 5701712-5701 712, 2013.

[23] K. Zhang, C. Mao, J. Lu, D. Wang, X. Chen, and J. Zhang, "Optimal control of state-of-charge of superconducting magnetic energy storage for wind power system," IET Renewable Power Generation, vol. 8, no. 1, pp. 58-66, 2014.

Received January 12 - 2016

Revised January 23 - 2017 